\title{
Spousal sexual violence, sexual behavior and sexually transmitted infections among ever- married women in Uganda
}

\author{
Stephen Ojiambo Wandera, James P.M. Ntozi and Betty Kwagala \\ Department of Department of Population Studies, \\ Institute of Statistics and Applied Economics, \\ Makerere University \\ swandera@gmail.com
}

\begin{abstract}
Despite the increasing recognition of the significance of spousal sexual violence in developing countries, evidence on its consequences for reproductive health remains limited. The aim of the paper was to examine the relationship between spousal sexual violence (SSV) and sexually transmitted infections (STIs) using a sample of 1749 ever-married women, from the 2006 Uganda Demographic and Health Survey. Pearson Chi-square tests and binary logistic regressions were used to investigate associations between SSV, STIs and selected reproductive health outcomes. From the analyses, $25 \%$ and $15 \%$ of ever-married women experienced SSV and reported STIs, respectively in the last 12 months. Women who experienced SSV were twice more likely to have had STIs in the last 12 months compared to those who did not. SSV is an important social and public health problem having implications on women's reproductive health and interventions to improve it should directly address the issue of spousal sexual violence.
\end{abstract}

Keywords and phrases: Spousal sexual violence, sexual behaviour and sexually transmitted infections

\section{Résumé}

Malgré la reconnaissance croissante de l'importance de la violence conjugale sexuelle dans les pays en développement, les données sur ses conséquences pour la santé de la procréation reste limité. Le but du document est d'examiner la relation entre la violence sexuelle conjugale (SSV) et les infections sexuellement transmissibles (IST) en utilisant un échantillon de 1749 femmes non mariées, de l'Ouganda 2006 Demographic and Health Survey. Pearson tests du chi carré et binaire régressions logistiques ont été utilisés pour étudier les associations entre les SSV, les IST et sélectionné les résultats de santé génésique. A partir des analyses, $25 \%$ et $15 \%$ des femmes mariées expérimentés SSV et IST rapportées respectivement au cours des 12 derniers mois. Les femmes victimes de SSV étaient deux fois plus susceptibles d'avoir eu des IST au cours des 12 derniers mois par rapport à ceux qui n'ont pas. SSV est un important problème social et de santé publique ayant des implications sur la santé reproductive des femmes et des interventions visant à améliorer II convient de s'attaquer directement au problème de la violence sexuelle conjugale. 
Les phrases-clés: Spousal violence sexuelle, les comportements sexuels et infections transmissibles sexuellement

\section{Introduction}

Spousal sexual violence (SSV) is the use of physical force to compel a spouse to engage in a sexual act against his or her will. It also means attempted or completed sexual act with a person who is unable to understand the nature of the act, decline participation and communicate unwillingness due to illness, disability, influence of alcohol, drugs and intimidation (Saltzman et al., 2002; Krug et al., 2002).

Spousal sexual violence is a global public health problem. Worldwide, nearly one in four women experience sexual violence by an intimate partner (Hakimi, 200I; Ellsberg, 1997; Mooney, 1993). Furthermore, one-third of adolescent girls report their first sexual experience as being forced (Jewkes, 200I; Matasha, 1998; Buga, et.al, 1996).

Studies in Mexico and the United States indicated that $40 \%$ and $52 \%$ respectively, of women experienced physical and sexual violence by their sexual partners. In addition, $23 \%$ of women in North London (England) and Nicaragua (21.7\%) reported sexual violence by a sexual partner in their lifetime (Campbell \& Soeken, 1999).

The proportion of ever-married women reporting spousal physical or sexual violence among developing countries was: Zambia (48\%), Colombia (44\%), Peru (42\%), Egypt (34\%), Nicaragua (30\%), Haiti (29\%), Dominican Republic (22\%), India (19\%) and Cambodia (18\%). In most countries, the highest rates of sexual violence occur in moderately wealthy house- holds, and not, as often assumed, among the poorest households (Kishor and Kiersten, 2004). In Zimbabwe and Ethiopia, $26 \%$ and $59 \%$ respectively, of ever-partnered women experienced spousal sexual violence (SSV), with $20 \%$ and $40 \%$ respectively reporting unwanted sex in the year before the survey (Watts, 1998; Gossaye, 2003).

In South Africa, 10\% of 15-19 year old women were forced to have sex against their will by their current or expartners, indicating that sexual violence was high among adolescent women (Department of Health et. al., 2002). In Kenya, $43 \%$ of 15-49 year old women reported lifetime experience of violence, with $16 \%$ reporting sexual violence (Askew and Ndhlovu, 2006). In Uganda, $36 \%$ of all women have experienced sexual violence and $30 \%$ of ever-married women have experienced SSV (UBOS and Macro International, 2007).

\section{Literature review and theoretical framework}

Spousal sexual violence has a direct impact on women's reproductive health. Previous studies demonstrate significant associations between spousal sexual violence and risky sexual behaviour among women. For example, sexually violated women are less likely to use condoms during intercourse because they are forced (Kiragu and Zabin, 1993; Koenig et. al., 2003). Several studies have found out that such women are more likely to have multiple sexual partners (Heise et al., 1993; Heise, 1995; Kishor and Kierstein, 
2004; Erulker, 2004; Olsson et al., 2000 and Abma, 1998). Worse still, sexually violated women are more likely to have sexually transmitted infection (STIs) as a result of risky sexual behaviors (Decker et al., 2005; Patel, 2005; Salam, 2006; Pamela et al., 1997; Dude, 2006; Martin, 1999).

Our hypotheses were: first, that women with multiple sexual partners are more likely to report having STIs than those with one partner or none. The second hypothesis is that women who do not use condoms during sexual intercourse are more likely to report having STls than those who use them. Thirdly, women who experienced spousal sexual violence are more likely to report STIs than those who did not.

Therefore, the aim of the paper was to examine the relationship between spousal sexual violence and sexually transmitted infections among ever-married women in Uganda. More specifically, the paper sought to:

I. Examine the association of background factors (of ever-married women's and their spouses') and spousal sexual violence;

2. Investigate the association between risky sexual behaviors and selfreported prevalence of STls among ever-married women; and

3. Study the relationship between spousal sexual violence and selfreported STIs among ever-married women in Uganda.

\section{Data and methods}

The paper used secondary data obtained from the Uganda Demographic and Health Survey (UDHS) of 2006. The survey investigated 2169 women using the Domestic Violence
Module, which asked questions on SSV and STIs. In the survey, 58 women were excluded for lack of privacy and 24 were not interviewed for other reasons leaving 2087 women. Of the 2087 women interviewed, 1749 ever-married women were selected from the main dataset for further analysis (UBOS and Macro International, 2007).

The main covariates are: spousal sexual violence and the three measures of risky sexual behavior: early sexual debut, non-condom use and multiple partnerships. Spousal sexual violence was measured by the question: Does / did your (last) husband / partner ever physically force you to have sexual intercourse with him even when you didn't want to? The responses were yes, no and don't know, which were recoded into binary form; $I=$ Yes and $0=$ No $/$ Don't Know, so that the reference category was women who had not been forced to have sex by their spouses.

In this paper, three measures of sexual risk behaviors were used as discussed below:

a. Early sexual debut - having sex before age 15. This was a dichotomous variable coded as I for those who had their first sexual experience prior to age 15 and 0 for those who were not yet sexually active or became sexually active at age 15 and beyond.

b. Having multiple partners - this was recorded as I for those who had had more than one sexual partner and 0 for those who had no partners or had strictly one partner and

c. Non-condom use at last sex - this was coded as I for those who did not use a condom at last sex and 0 
for those who had never had sex or who had used a condom at last sex. Other independent variables included women's background characteristics (age, marital status, wealth index, education, region, occupation and residence) and those of their spouses (age, education, occupation and alcohol consumption).

The dependent variable in this paper is self - reported STIs. The UDHS of 2006 measured STI and symptomatic self-reporting among ever-married women using the following question: have you had a genital ulcer or sore within the past 12 months? The responses to this question were binary in nature (Yes $=\mathrm{I}$ and a $\mathrm{No}=0$ ).

Data analysis softwares included SPSS (Version I5) and STATA (version 10). Data analyses were done at three levels: univariate, bivariate and multivariate analyses. At univariate level, frequency distributions were made to describe women's background characteristics and those of their partners, the prevalence of risky sexual behaviors, spousal sexual violence and selfreported STIs. Cross tabulations were run for the bivariate analysis and Pearson chi-square tests were used to explain the association between: background (of women and their spouses) factors with spousal sexual violence; risky sexual behaviors, spousal sexual violence and STIs. A " $p$ " value less than or equal to 0.050 , connotes a statistically significant association between the independent and dependent variables.
Multivariate analysis measured the net effect of the explanatory factors using binary logistic regression on the dependent variable (self-reported STIs). The strengths of relationships are reported as odds ratios (ORs). Dummy variables were created for each of the categorical explanatory variables for use in multivariate analysis. Reference categories took the value of 0 and categories of interest were $I$.

\section{Results}

Background characteristics of the
respondents and their spouses

Table I shows that the majority of the respondents (22\%) were aged 25-29 years and few $(6 \%)$ of them were adolescents aged 15-19 years. Most of the women $(86 \%)$ were rural residents and $(60 \%)$ had attained primary education. The highest proportion $(23 \%)$ of the women was from the poorest wealth index $(23 \%)$ and the least from the middle class (17\%).

In general, most of the respondents (65\%) were married, a high proportion $(70 \%)$ was involved in agriculture and the majority $(29 \%)$ was from the northern region.

The distribution of the spouses of the respondents is also displayed in Table I. The majority (39\%) of them were aged $25-34$ and very few $(6 \%)$ were above 55 years. Most (58\%) of the spouses of these women had primary education, $57 \%$ were involved in agriculture and $57 \%$ consumed alcohol. 
Table I: Percent distribution of ever-married women and their spouses by background characteristics and alcohol consumption

\begin{tabular}{|c|c|c|}
\hline Characteristics & $\begin{array}{c}\text { Frequency }(\mathrm{N}) \\
\text { (Ever-married women) }\end{array}$ & Percent (\%) \\
\hline \multicolumn{3}{|c|}{ Age group } \\
\hline $15-19$ & 110 & 6.3 \\
\hline $20-24$ & 367 & 21 \\
\hline $25-29$ & 384 & 22 \\
\hline $30-34$ & 335 & 19.2 \\
\hline $35-39$ & 250 & 14.3 \\
\hline $40-44$ & 168 & 9.6 \\
\hline $45-49$ & 135 & 7.7 \\
\hline \multicolumn{3}{|c|}{ Residence } \\
\hline Urban & 249 & 14.2 \\
\hline Rural & 1500 & 85.8 \\
\hline \multicolumn{3}{|c|}{ Education } \\
\hline No education & 435 & 24.9 \\
\hline Primary & 1055 & 60.3 \\
\hline Secondary & 210 & 12.0 \\
\hline Higher & 49 & 2.8 \\
\hline \multicolumn{3}{|c|}{ Wealth index } \\
\hline Poorest & 402 & 23.0 \\
\hline Poorer & 373 & 21.3 \\
\hline Middle & 305 & 17.4 \\
\hline Richer & 320 & 18.3 \\
\hline Richest & 349 & 20.0 \\
\hline \multicolumn{3}{|c|}{ Marital status } \\
\hline Married & 1142 & 65.3 \\
\hline Living together & 340 & 19.4 \\
\hline Widowed & 85 & 4.9 \\
\hline Divorced & 23 & 1.3 \\
\hline Separated & 159 & 9.1 \\
\hline \multicolumn{3}{|c|}{ Region } \\
\hline Central & 476 & 27.2 \\
\hline Eastern & 388 & 22.2 \\
\hline Northern & 507 & 29.0 \\
\hline Western & 378 & 21.6 \\
\hline \multicolumn{3}{|c|}{ Occupation } \\
\hline Not working & 138 & 7.9 \\
\hline Professional & 130 & 7.4 \\
\hline
\end{tabular}




\begin{tabular}{|c|c|c|}
\hline Sales & $|5|$ & 8.6 \\
\hline Agriculture & 1228 & 70.2 \\
\hline Manual & 102 & 5.8 \\
\hline Total & 1749 & 100 \\
\hline Characteristics & Frequency $(\mathrm{N})$ & Percent (\%) \\
\hline \multicolumn{3}{|c|}{ (Spouses of ever-married women) } \\
\hline \multicolumn{3}{|c|}{ Age } \\
\hline $15-24$ & 135 & 9.1 \\
\hline $25-34$ & 572 & 38.7 \\
\hline $35-44$ & 462 & 31.2 \\
\hline $45-54$ & 223 & 15.1 \\
\hline $55+$ & 87 & 5.9 \\
\hline \multicolumn{3}{|c|}{ Partner's education } \\
\hline No education & 255 & 14.6 \\
\hline Primary & 1005 & 57.5 \\
\hline Secondary & 375 & 21.4 \\
\hline Higher & 114 & 6.5 \\
\hline \multicolumn{3}{|c|}{ Partner's occupation } \\
\hline Did not work & 106 & 6.1 \\
\hline Professional work & 126 & 7.2 \\
\hline Sales & 194 & II.I \\
\hline Agriculture & 992 & 56.7 \\
\hline Services & 13 & 0.7 \\
\hline Manual & 318 & 18.2 \\
\hline \multicolumn{3}{|c|}{ Partner drinks alcohol } \\
\hline No & 753 & 43.1 \\
\hline Yes & 992 & 56.7 \\
\hline Total & 1749 & 100 \\
\hline
\end{tabular}

Ever-married women's experience

of spousal sexual violence by background factors

Table 2 shows that, over all, one out of every four $(25 \%)$ ever-married women experienced spousal sexual violence in the last 12 months and this violence varied with women's background factors.

A higher proportion (26\%) of rural women than urban ones (19\%) experienced spousal sexual violence. The association between residence and SSV was statistically significant $(p=0.027)$.

The experience of SSV was highest $(38 \%)$ in eastern region followed by western $(27 \%)$, central $(23 \%)$ and lowest $(15 \%)$ in the northern region. The association between region and SSV was highly significant $(p=0.000)$.

Women's education had a negative association with their experience of SSV. SSV was highest (27\%) among women with no education and least (8\%) among those with higher education. The association between educa- 
tion and SSV was significant $(p=0.038)$.

Spousal sexual violence was highest (33\%) among the middle class women and least (20\%) among the richest women. Women's wealth index had a significant association with spousal sex- ual violence $(p=0.002)$.

Table 2 also shows that age $(0.547)$, marital status (0.058) and occupation (0.007) of ever-married women were not significantly associated with SSV.

Table 2: Percentage of ever-married women who experienced spousal sexual violence in the last 12 months, by background factors

\begin{tabular}{|c|c|c|c|c|}
\hline \multicolumn{5}{|c|}{ Experienced spousal sexual violence in the last 12 months } \\
\hline Variables & No (\%) & Yes (\%) & $\mathrm{N}$ & $\mathrm{p}$-value \\
\hline \multicolumn{5}{|c|}{ Age } \\
\hline $15-19$ & 74.55 & 25.45 & 110 & \\
\hline $20-24$ & 75.75 & 24.25 & 367 & \\
\hline $25-29$ & 76.30 & 23.70 & 384 & \\
\hline $30-34$ & 72.24 & 27.76 & 335 & 0.547 \\
\hline $35-39$ & 72.80 & 27.20 & 250 & \\
\hline $40-44$ & 76.19 & 23.81 & 168 & \\
\hline $45-49$ & 80.74 & 19.26 & 135 & \\
\hline \multicolumn{5}{|c|}{ Residence } \\
\hline Urban & 80.7 & 19.3 & 249 & 0.027 \\
\hline Rural & 74.2 & 25.8 & 1500 & \\
\hline \multicolumn{5}{|c|}{ Region } \\
\hline Central & 77.3 & 22.7 & 476 & \\
\hline Eastern & 61.9 & 38.1 & 388 & 0.000 \\
\hline Northern & 84.6 & 15.4 & 507 & \\
\hline Western & 73.5 & 26.5 & 378 & \\
\hline \multicolumn{5}{|c|}{ Education } \\
\hline No education & 73.1 & 26.9 & 435 & \\
\hline Primary & 75.0 & 25.0 & 1055 & 0.038 \\
\hline Secondary & 76.2 & 23.8 & 210 & \\
\hline Higher & 91.8 & 8.2 & 49 & \\
\hline \multicolumn{5}{|c|}{ Wealth index } \\
\hline Poorest & 77.6 & 22.4 & 402 & \\
\hline Poorer & 75.6 & 24.4 & 373 & 0.002 \\
\hline Middle & 67.2 & 32.8 & 305 & \\
\hline Richer & 73.4 & 26.6 & 320 & \\
\hline Richest & 80.2 & 19.8 & 349 & \\
\hline \multicolumn{5}{|c|}{ Current marital status } \\
\hline Married & 75.8 & 24.2 & 1142 & \\
\hline Living together & 69.4 & 30.6 & 340 & 0.058 \\
\hline Widowed & 77.6 & 22.4 & 85 & \\
\hline
\end{tabular}




\begin{tabular}{lcccc} 
Divorced & 78.3 & 21.7 & 23 & \\
Separated & 80.5 & 19.5 & 159 & \\
\hline Not working & \multicolumn{3}{c}{ Occupation } & \\
Professional & 83.3 & 16.7 & 138 & 0.070 \\
Sales & 80.8 & 19.2 & 130 & \\
Agriculture & 75.5 & 24.5 & 151 & \\
Manual & 73.6 & 26.4 & 1228 & \\
Total numbers and \% & 75.5 & 24.5 & 102 & \\
\hline
\end{tabular}

Table 3: Percentage of ever-married women who experienced SSV in the last 12 months, by male spouses' background factors and alcohol consumption

\begin{tabular}{|c|c|c|c|c|}
\hline \multirow[t]{2}{*}{ Variables } & \multicolumn{4}{|c|}{ Experienced spousal sexual violence in the last 12 months } \\
\hline & No (\%) & Yes (\%) & $\mathrm{N}$ & $\mathrm{p}$-value \\
\hline \multicolumn{5}{|c|}{ Age } \\
\hline $15-24$ & 80.0 & 20.0 & 135 & \\
\hline $25-34$ & 73.3 & 26.7 & 572 & 0.550 \\
\hline $35-44$ & 74.0 & 26.0 & 462 & \\
\hline $45-54$ & 73.5 & 26.5 & 223 & \\
\hline $55+$ & 77.0 & 23.0 & 87 & \\
\hline \multicolumn{5}{|c|}{ Partner's education } \\
\hline No education & 77.3 & 22.7 & 255 & \\
\hline Primary & 72.4 & 27.6 & 1005 & 0.003 \\
\hline Secondary & 77.3 & 22.7 & 375 & \\
\hline Higher & 86.8 & 13.2 & 114 & \\
\hline \multicolumn{5}{|c|}{ Partner's occupation } \\
\hline Did not work & 81.1 & 18.9 & 106 & \\
\hline Professional work & 84.1 & 15.9 & 126 & 0.015 \\
\hline Sales & 76.3 & 23.7 & 194 & \\
\hline Agriculture & 73.3 & 26.7 & 992 & \\
\hline Services & 100 & 0 & 13 & \\
\hline Manual & 73.6 & 26.4 & 318 & \\
\hline \multicolumn{5}{|c|}{ Partner drinks alcohol } \\
\hline No & 77.6 & 22.4 & 753 & 0.046 \\
\hline Yes & 73.4 & 26.6 & 992 & \\
\hline Total & 1315 & 434 & 1749 & \\
\hline
\end{tabular}


Ever-married women's experience of spousal sexual violence by spouses' background factors and alcohol consumption

Table 3 presents the results of crosstabulations of spousal sexual violence and spouses' background factors and alcohol consumption. Spouses' age $(p=0.55)$ was not significantly associated with SSV.

Spouses with primary education sexually violated their women most ( $28 \%)$, followed by those with no education $(23 \%)$ and least by spouses with higher education (13\%). The spouses' education was significantly associated with spousal sexual violence $(p=0.003)$.

Spouses involved in agriculture sexually violated their partners most (27\%) followed by those in manual labors (26\%) and in sales (24\%) although few women whose partners were involved in services had no experience of sexual violence at all (0\%). The spouses' occupation was significantly associated with women's experience of sexual violence $(p=0.0015)$.

It is notable that men who drink alcohol violated their sexual partners (27\%) more than those who did not drink alcohol. Drinking alcohol by spouses was significantly associated with the women's experience of sexual violence $(p=0.046)$.

Ever-married women's risky sexual behavior and STIs

The distribution of ever-married women's risky sexual behaviour and their cross-tabulations with selfreported STls are presented in Table 4 and 5 respectively. From Table 4, most women (55\%) had multiple sexual partners, $85 \%$ did not use condoms during last sexual intercourse and $20 \%$ had first sex before age 15 .

Nearly two out of every ten (I5\%) ever-married women reported an STI in the last 12 months before the survey. Similarly, one out of every four $(25 \%)$ ever-married women experienced SSV in the last 12 months preceding the survey (Table 4).

In Table 5, it can be seen that selfreported STls were highest (19\%) among ever-married women who had first sex before age 15 and lowest (14\%) among those who had sex later than 15 years. First sexual experiences among younger women ( $<15$ years) are usually forced and associated with non-condom use, which predisposes them to STIs. The association of early sexual debut and STI was statistically significant $(p=0.006)$.

Women in multiple partnerships were twice $(20 \%)$ more likely to report STIs compared to those who had one partner only or none (9\%). Having multiple partners (with inconsistent or noncondom use) directly predisposes women to STIs. There was a highly significant association between multiple partnering and self-reported STIS $(p=0.000)$.

Table 5 further shows that the prevalence of self-reported STIs was nearly twice as high (23\%) among ever-married women who experienced spousal sexual violence compared to those who had not (I2\%). The association between SSV and STIs was statistically significant $(p=0.000)$. In contrast, the association between condom use and self-reported STls was not significant $(p=0.45)$. This is because a one time, other than consistent and proper condom use, cannot prevent STIs. 
Table 4: Percentage distribution of ever-married women by risky sexual behaviour, SSV and STIs

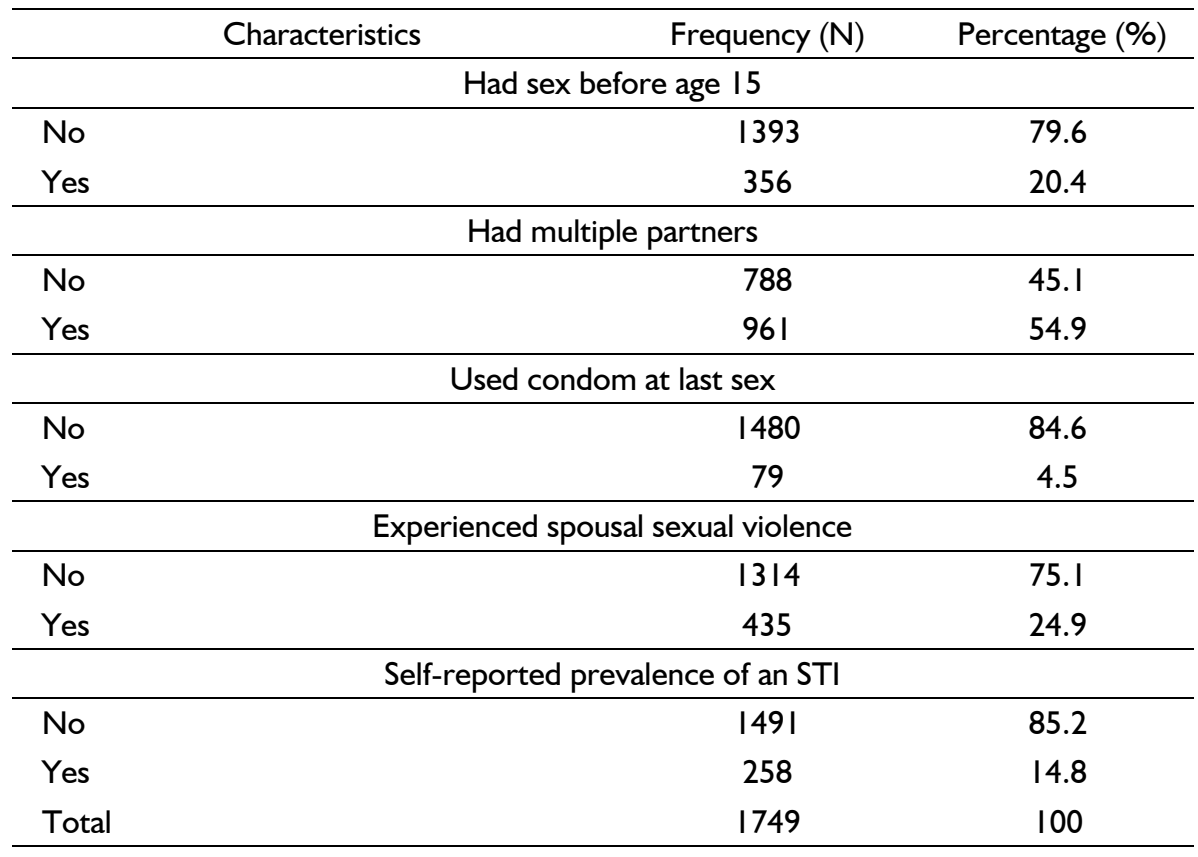

Table 5: Associations between women's risky sexual behaviors, SSV and STIs

\begin{tabular}{|c|c|c|c|c|}
\hline & \multicolumn{4}{|c|}{ Had an STI in the last 12 months } \\
\hline & No (\%) & Yes (\%) & $\mathrm{N}$ & $\mathrm{p}$-value \\
\hline \multicolumn{5}{|c|}{ Had sex before age 15} \\
\hline No & 86.4 & 13.6 & 1393 & 0.006 \\
\hline Yes & 80.6 & 19.4 & 356 & \\
\hline \multicolumn{5}{|c|}{ Used condom at last sex } \\
\hline No & 84.2 & 15.8 & 1480 & 0.452 \\
\hline Yes & 87.3 & 12.7 & 79 & \\
\hline \multicolumn{5}{|c|}{ Had multiple partners } \\
\hline No & 91.1 & 8.9 & 788 & 0.000 \\
\hline Yes & 80.4 & 19.6 & 961 & \\
\hline \multicolumn{5}{|c|}{ Experienced spousal sexual violence } \\
\hline No & 88.0 & 12.0 & 1314 & 0.000 \\
\hline Yes & 77.0 & 23.0 & 435 & \\
\hline Total & |49| & 258 & 1749 & \\
\hline
\end{tabular}

Multivariate analysis results

In order to establish the significant predictors of sexually transmitted infec- tions among ever-married women in the last 12 months, a logistic regression model was fitted and the results are 
shown in Table 6. The prevalence of self-reported STIs among ever-married women significantly depended on women's marital status, region, noncondom use, multiple partnerships and spousal sexual violence.

Regarding women's marital status, widowed women significantly had decreased odds of self-reported prevalence of STIs (OR = 0.296) compared to separated or divorced women. This relationship was statistically significant $(p=0.020)$. However, the experience of STls did not significantly vary among married and cohabiting women compared to those separated or divorced.

More interestingly, ever-married women's region was a very significant predictor of self-reported STIs. Compared with women from northern region, those from central region were four times $(O R=4.113)$ more likely to report having STIs in the last 12 months and this relationship was statistically significant $(p=0.000)$. Similarly, women from eastern region, compared to those from northern region, were thrice $(O R=3.014)$ more likely to report having STIs in the last 12 months. This relationship was highly significant $(p=0.000)$. Furthermore, women from western region were more than twice $(O R=2.483)$ more likely to report having STIs in the last 12 months compared to those from northern region and the relationship was also statistically significant $(p=0.00 \mathrm{I})$.

Of the three risky sexual behaviours, non-condom use and having multiple partners had significant relationships with the experience of STIs among ever-married women. The odds of experiencing STls were higher (almost twice) (OR = I.9) among evermarried women who did not use condoms during last sex, than among those who used condoms during last sex and this relationship was significant $(p=$ 0.022). In addition, women who had multiple partners, compared to those who had one or none, were nearly twice $(O R=1.8)$ more likely to report having STIs in the last 12 months and the relationship was statistically significant $(p=0.00 I)$ as shown in Table 7 .

Furthermore, spousal sexual violence is a significant factor for selfreported prevalence of STls among ever-married women in Uganda. The self-reported prevalence of STls among ever-married women who had experienced spousal sexual violence is nearly twice that among women who had not experienced such violence in the last 12 months $(O R=1.9)$. This relationship was highly statistically significant $(p-$ value 0.000 ). 
Table 6: Adjusted odds ratios from logistic regression model examining the effect of spousal sexual violence on self-reported STIs in the last I 2 months, controlling for background factors and risky sexual behaviors

\begin{tabular}{|c|c|c|c|}
\hline Background factors & Odds ratios (OR) & $\begin{array}{l}\text { Standard } \\
\text { errors }\end{array}$ & $\mathrm{p}$ - value \\
\hline \multicolumn{4}{|c|}{ Women's age } \\
\hline $15-24$ & 0.883 & 0.221 & 0.620 \\
\hline $25-34$ & 1.179 & 0.217 & 0.373 \\
\hline $35+(R)$ & 1.000 & & \\
\hline \multicolumn{4}{|c|}{ Women's education level } \\
\hline None $(\mathrm{R})$ & 1.000 & & \\
\hline Primary & 1.446 & 0.302 & 0.078 \\
\hline Higher & 1.469 & 0.433 & 0.192 \\
\hline \multicolumn{4}{|c|}{ Women's wealth index } \\
\hline Poor (R) & 1.000 & & \\
\hline Middle & 1.168 & 0.261 & 0.489 \\
\hline Rich & 1.468 & 0.307 & 0.066 \\
\hline \multicolumn{4}{|c|}{ Women's marital status } \\
\hline Married & 0.803 & 0.213 & 0.409 \\
\hline Cohabiting & 0.774 & 0.229 & 0.387 \\
\hline Widowed & 0.296 & 0.155 & $0.020^{*}$ \\
\hline Separated/Divorced(R) & 1.000 & & \\
\hline \multicolumn{4}{|c|}{ Women's residence } \\
\hline Rural (R) & 1.000 & & \\
\hline Urban & 1.046 & 0.247 & 0.847 \\
\hline \multicolumn{4}{|l|}{ Women's region } \\
\hline Central & 4.113 & 1.160 & $0.000^{* * * *}$ \\
\hline Eastern & 3.014 & 0.831 & $0.000^{* * * *}$ \\
\hline Western & 2.483 & 0.706 & $0.001^{* *}$ \\
\hline Northern (R) & 1.000 & & \\
\hline \multicolumn{4}{|c|}{ Women's occupation } \\
\hline Professional & 0.656 & 0.232 & 0.234 \\
\hline Sales and services & 0.978 & 0.326 & 0.947 \\
\hline Agriculture & 1.169 & 0.343 & 0.593 \\
\hline Manual & 1.293 & 0.507 & 0.511 \\
\hline Not working $(\mathrm{R})$ & 1.000 & & \\
\hline \multicolumn{4}{|c|}{${ }^{*} \mathrm{p}<0.05,{ }^{*} * \mathrm{p}<0.0 \mathrm{I}$ and ${ }^{*} * * \mathrm{p}<0.00 \mathrm{I},(\mathrm{R})-$ Reference Category } \\
\hline Background factors & Odds Ratios (OR) & $\begin{array}{c}\text { Standard } \\
\text { Errors }\end{array}$ & $\mathrm{p}$ - value \\
\hline \multicolumn{4}{|c|}{ Partner's Age } \\
\hline $15-24$ & 0.637 & 0.242 & 0.237 \\
\hline
\end{tabular}




\begin{tabular}{|c|c|c|c|}
\hline $25-34$ & 0.880 & 0.170 & 0.512 \\
\hline $35+(R)$ & 1.000 & & \\
\hline \multicolumn{4}{|c|}{ Partner's education level } \\
\hline Primary & 0.762 & 0.175 & 0.239 \\
\hline Secondary & 0.749 & 0.201 & 0.281 \\
\hline Higher & 0.713 & 0.283 & 0.395 \\
\hline No education (R) & 1.000 & & \\
\hline \multicolumn{4}{|c|}{ Partner's occupation } \\
\hline Professional & 3.190 & 3.429 & 0.281 \\
\hline Sales \& services & 2.716 & 2.930 & 0.354 \\
\hline Agriculture & 2.918 & 3.120 & 0.317 \\
\hline Manual & 3.580 & 3.820 & 0.232 \\
\hline Did not work (R) & 1.000 & & \\
\hline \multicolumn{4}{|c|}{ Partner drinks alcohol } \\
\hline No $(R)$ & 1.000 & & \\
\hline Yes & 1.290 & 0.190 & 0.085 \\
\hline \multicolumn{4}{|c|}{ Women used condom during last sex } \\
\hline Yes (R) & 1.000 & & \\
\hline No & 1.885 & 0.521 & $0.022^{*}$ \\
\hline \multicolumn{4}{|c|}{ Women had sex before age 15} \\
\hline Yes & 1.160 & 0.200 & 0.389 \\
\hline No $(R)$ & 1.000 & & \\
\hline \multicolumn{4}{|c|}{ Women had multiple partners } \\
\hline Yes & 1.787 & 0.303 & $0.001^{* *}$ \\
\hline No $(R)$ & 1.000 & & \\
\hline \multicolumn{4}{|c|}{ Women experienced spousal sexual violence } \\
\hline Yes & 1.878 & 0.291 & $0.000^{* * * *}$ \\
\hline No $(R)$ & 1.000 & & \\
\hline
\end{tabular}

\begin{tabular}{lccc}
\hline Background factors & Odds ratios (OR) & $\begin{array}{c}\text { Standard } \\
\text { errors }\end{array}$ & $\mathrm{p}$ - value \\
\hline \multicolumn{4}{c}{ Partner's age } \\
$25-34$ & 0.637 & 0.242 & 0.237 \\
$35+(\mathrm{R})$ & 0.880 & 0.170 & 0.512 \\
\hline
\end{tabular}

\begin{tabular}{lccc}
\hline \multicolumn{4}{c}{ Partner's education level } \\
\hline Primary & 0.762 & 0.175 & 0.239 \\
Secondary & 0.749 & 0.201 & 0.281 \\
Higher & 0.713 & 0.283 & 0.395
\end{tabular}




\begin{tabular}{|c|c|c|c|}
\hline No education (R) & 1.000 & & \\
\hline \multicolumn{4}{|c|}{ Partner's occupation } \\
\hline Professional & 3.190 & 3.429 & 0.281 \\
\hline Sales \& services & 2.716 & 2.930 & 0.354 \\
\hline Agriculture & 2.918 & 3.120 & 0.317 \\
\hline Manual & 3.580 & 3.820 & 0.232 \\
\hline Did not work (R) & 1.000 & & \\
\hline \multicolumn{4}{|c|}{ Partner drinks alcohol } \\
\hline No $(R)$ & 1.000 & & \\
\hline Yes & 1.290 & 0.190 & 0.085 \\
\hline \multicolumn{4}{|c|}{ Women used condom during last sex } \\
\hline Yes (R) & 1.000 & & \\
\hline No & 1.885 & 0.521 & $0.022^{*}$ \\
\hline \multicolumn{4}{|c|}{ Women had sex before age I5 } \\
\hline Yes & 1.160 & 0.200 & 0.389 \\
\hline No $(R)$ & 1.000 & & \\
\hline \multicolumn{4}{|c|}{ Women had multiple partners } \\
\hline Yes & I.787 & 0.303 & $0.001^{* * *}$ \\
\hline No $(R)$ & 1.000 & & \\
\hline \multicolumn{4}{|c|}{ Women experienced spousal sexual violence } \\
\hline Yes & 1.878 & 0.291 & $0.000^{* * * *}$ \\
\hline No (R) & 1.000 & & \\
\hline
\end{tabular}

\section{Discussion}

From the analysis, one in every four ever-married women (25\%) experienced spousal sexual violence in the last 12-months. This result is in consonance with other studies which revealed high prevalence of spousal sexual violence (Askew and Ndhlovu, 2006; Gossaye, 2003; Central Statistical Office et al., 2002). On the other hand, nearly two out of every ten (15\%) ever-married women reported ever having an STI in the last 12 months preceding the survey. In addition, most women (55\%) had multiple sexual partners, $85 \%$ did not use condoms at last sex and $20 \%$ had first sex before age 15.

At a bivariate level of analysis, the factors that were significantly associ- ated with spousal sexual violence were women's residence, region, education, occupation and wealth index and spouses' education and occupation. Spousal sexual violence was more prevalent among women from eastern region and from middle wealth index. Since alcohol consumption is highest in eastern region especially in the Teso region, it probably accounts for the highest reported SSV. It is interesting to note that middle class women experienced more SSV than any other category. This pattern is similar to the inverted $U$ shape found by Kishor and Kiersten (2004) in a multi-country study on Cambodia, Colombia, Egypt and other developing countries, with violence peaking among middle wealth 
quartile women.

The significant predictors of selfreported STls were women's marital status, region, risky sexual behaviours (non-condom use and multiple partnerships) and spousal sexual violence. Women from central, eastern and western regions were several times $(\mathrm{OR}=4 . \mathrm{II}, 3.0 \mathrm{I}$ and 2.50 respectively) more likely to report having STIs in the last 12 months, compared to those from northern Uganda. This could be because of the high prevalence of SSV in these regions $(38 \%$ in eastern, $27 \%$ in western and $23 \%$ in central) compared to northern region (15\%). SSV has direct and indirect implications on sexual behaviour and STIs (Decker et al., 2005; Patel, 2005; Salam, 2006; Pamela et al., 1997; Dude, 2006; Martin et al., 1999; Greenberg, 1999). In addition, women from the three regions engage more in risky sexual behaviours (especially multiple sexual partnerships) than those from northern region.

Women who were sexually violated by their spouses were nearly twice $(O R=1.9)$ more likely to experience STls in the last 12 months. This finding is in consonance with several studies that have found a similar relationship between spousal sexual violence and STIs among women (Decker, et al., 2005; Koenig et al., 2003; Martin et al., 1998; Erulker, 2004; Olsson et al., 2000; Senn et al., 2006; Erulker, 2004). This is because sexual violence diminishes the victim's sense of self-esteem, motivation for and power to negotiate for protected sex with her spouse, which increases the risk of contracting STIs (Finklehor and Browne, 1985; Wieland, 1997; Koenig et al., 2003 \& Maman et al., 2000). In addition, spousal sexual violence increases the odds of having multiple partners among women (Heise et al., 1993; Heise, 1995; Kishor et al., 2004).

As shown in Table 7, the odds of experiencing STls were almost twice as high among ever-married women who did not use condoms during last sex $(\mathrm{OR}=1.9)$, compared to those who used condoms during last sex. Related to this, women who had multiple partners, compared to those who had one or none, were nearly twice more likely to report having STls in the last 12 months $(\mathrm{OR}=\mathrm{I} .8)$.

However, several limitations of the study merit our discussion. The first challenge is underreporting of sexual violence and self-reported STls among ever-married women. Sexual violence is a private matter, especially among married women, while STls are highly stigmatizing in the community. Secondly, the paper is limited by inadequate knowledge of respondents about STI symptoms especially among uneducated women. This could have led to underestimation of the prevalence of STls among ever-married women. Actually, the correlation between selfreported STIs and clinically identified or laboratory-confirmed STls has been shown to be quite low even though, the former are useful in assessing women's reproductive behaviour and outcomes.

Finally, the paper is unable to assume causality in the relationship between spousal sexual violence and self-reported STI. Besides, one cannot assert that self reported STls were a consequence of sexual violence since timing of the STIs and violence are not measured in UDHS. 


\section{Conclusion}

Based on these findings, the paper recommends several interventions: first, STIs screening and treatment (Management) programmes targeting central, eastern and western regions. In this paper, these regions had increased odds of STls among ever-married women. The intervention can involve community sensitization, training of service providers in health centres and integration of STI management into health service's provision.

Secondly, it is important to strengthen the $A B C C^{\prime}$ strategy through massive behaviour change communication (BCC) programmes. Information, Education and Communication (IEC) programmes can help promote faithfulness among married couples, which in turn lessens risky behaviour such as having multiple partners among married women. Encouraging condom use and male circumcision can also help reduce risks of STls among married people.

Thirdly, medico-legal response to sexual violence is vital for preventing STIs among married women. Medical response through post-exposure prophylaxis (PEP) within 32 hours of experience of sexual violence can reduce the odds of STIs among married women experiencing marital rape. Legal response by reporting extreme cases such as marital rape to police can reduce the continuity of sexual violence, which is a significant predictor of STIs among ever-married women especially in eastern and western Uganda.

In addition, it is necessary to promote initiatives that are aimed at preventing sexual and gender-based violence (SGBV) in Uganda. We need programmes designed to prevent SGBV in Eastern Uganda, which was found to be the leading region with SSV. The findings of the paper strongly suggest that SSV has major adverse consequences on women's sexual and reproductive health hence the need to integrate management of SGBV component into current reproductive health service programs.

In the future, investigating the relationship between spousal sexual violence and HIV/AIDS status of women would be a potential paper area. There is need for further research on the relationship between sexual violence and incidence of STls among women using clinical approaches. It is necessary to explore the reason(s) why SSV is most prevalent among middle class women and in Eastern Uganda.

\section{References}

Abma, J., Driscoll, A. and Moore, K. 1998. Young women's degree of control over first intercourse: an exploratory analysis. Family Planning Perspectives, 30(I), pp. I2-I8.

Askew, L. and Ndhlovu, L. 2006. Developing a multisectoral and comprehensive response to Sexual and Gender Based Violence in East and Southern Africa. Project proposal to Swedish International Development Assistance from Population Council.

Buga, G.A., Amoko, D.H., Ncayiyana, D.J. 1996. Sexual behaviour, contraceptive practice and reproductive health among school adolescents in rural Transkei. South

I. $A B C C$ (Abstinence, Being Faithful to one partner, Condom use and Circumcision (Male)) is a strategy of preventing HIV/AIDS and other STIs in Uganda 
African Medical Journal, 86, pp. 523527.

Campbell, J.C. and Soeken, K.L. 1999. Forced sex and intimate partner violence: effects on women's risk and women's health. Violence against Women, 5, pp. 1017-1035.

Decker, M.R., Silverman, J.G. and Raj, A. 2005. Dating violence and Sexually Transmitted Disease / HIV testing and diagnosis among adolescent females. Pediatrics, I16, pp. 272-276.

Department of Health, Medical Research Council (MRC) and Measure DHS+. 2002. South Africa Demographic and Health Survey 1998, Full report. Pretoria: Department of Health.

Dude, A. 2006. Intimate partner violence and increased lifetime risk of sexually transmitted infections among women in Ukraine. Studies in Family Planning, (2), pp. 89-100.

Ellsberg, M.C. 1997. Candies in Hell: Domestic Violence against women in Nicaragua. Umea, Umea University.

Erulker, S.A. 2004. The Experience of Sexual Coercion Among Young People in Kenya. Family Planning Perspectives, 30 (4), pp. 15-24.

Finklehor, D and Browne, A. 1985. The Traumatic Impact of Child Sexual Abuse: A Conceptualization. Journal of Orthopsychiatry, 55, pp. 530-54I.

Gossaye, Y. 2003. Women's Health and Life Events Paper in rural Ethiopia. Ethiopian Journal of Health Development, 17 (2), pp. 2-50.

Hakimi, M. 200I. Silence for the sake of harmony: Domestic violence and women's health in central Java. Yogyakarta, Gadjah Mada University.
Heise, L. 1993. Violence against women: the hidden health burden. World Health Stat Q, 46(I), pp. 7885.

Heise, L., K. Moore, K. and Toubia, N. 1995. Sexual coercion and reproductive health: A focus on research. New York: The Population Council.

Heise, L.L. 1993. Reproductive freedom and violence against women: Where are the intersections? Journal of Law, Medicine and Ethics, 2I(2), pp. 206216.

Jewkes, R. 200I. Relationship dynamics and adolescent pregnancy in South Africa. Social Science and Medicine, 5, pp. 733-744.

Kiragu, K. and Zabin, L. 1993. Contraceptive use among high school students in Kenya. International Family Planning Perspectives, 21 .

Kishor, S. and Kiersten, J. 2004. Profiling Domestic Violence: A multi-country paper. Calverton, Maryland, USA: ORC Macro.

Koenig, M.A. Lutalo, T., Zhao, F., Nalugoda, F., Wabwire-Mangen, F., Kiwanuka, N., Wagman, J., Serwadda, D., Wawer, M and Gray, R. 2003. Domestic violence in rural Uganda: evidence from a community-based study. Bull World Health Organ, 8I (I), pp. 53-60.

Krug, E.G. L., Dahlberg, J., Mercy, A. and R. Lozano. 2002. World report on violence and health. World Health Organisation, Geneva.

Maman, S., Campbell, J., Sweat, M., \& Gielen, A. 2000. The intersections of HIV and violence: Directions for future research and interventions. 
Social Science and Medicine, 50, pp. 459-478.

Martin, S.L. 1999. Sexual behaviour and reproductive health outcomes: associations with wife abuse in India. Journal of the American Medical Association, 282, pp. 1967-1972.

Martin, S.L. 1999. Domestic violence and sexually transmitted diseases: the experience of prenatal care patients. Public Health Reports, I I4(3), pp. 262-268.

Martin, S.L. 1998. Women in prenatal care/substance abuse treatment program: links between domestic violence and mental health. Maternal and Child Health, 2(2), pp. 85-94.

Matasha, E. 1998. Sexual and reproductive health among primary and secondary school pupils in Mwanza, Tanzania: need for intervention. AIDS Care, 10, pp. 57I582.

Mooney, J. 1993. The hidden figure: domestic violence in north London. London, Middlesex University.

Olsson, A., Ellsberg, M., Berglund, S., Herrera, A., Zelaya, E. and Pena, R. 2000. Sexual abuse during childhood and adolescence among Nicaraguan men and women: A populationbased anonymous survey. Child Abuse and Neglect, 24, pp. 15791589.

Pamela, J., Wendy, L. and Hellerstedt, K. 1997. Current or Past Physical or Sexual Abuse as a Risk Marker for Sexually Transmitted Disease in
Pregnant Women. American Journal of Public Health, 87(6), pp. 10161018

Patel, V., Kirkwood, BR. and Weiss, H. 2005. Chronic fatigue in developing countries: population based survey of women in India. BMJ, 330, pp. 1190.

Salam, M.A., Alim, M.A. and Noguchi, T. 2006. Spousal abuse against women and its consequences on reproductive health: a study in the urban slums in Bangladesh. Maternal and Child Health Journal, 10, pp. 8394.

Saltzman, L.E., Fanslow, J.L., McMahon, P.M. and Shelley, G.A. 2002. Intimate partner violence surveillance: uniform definitions and recommended data elements, version 1.0. Atlanta (GA): Centers for Disease Control and Prevention, National Center for Injury Prevention and Control.

Uganda Bureau of Statistics (UBOS) and Macro International Inc. (2006). Uganda Demographic and Health Survey. Calverton, Maryland, USA: UBOS and Macro International Inc.

Watts, C. 1998. Withholding of sex and forced sex: dimensions of violence against Zimbabwean women. Reproductive Health Matters, 6(I2), pp. 57-65.

Wieland, S. 1997. Hearing the internal trauma: Working with children and adolescents who have been abused. Thousand Oaks, CA: Sage. 